\title{
Endoplasmic reticulum-mitochondria crosstalk in NIX-mediated murine cell death
}

\author{
Abhinav Diwan, ${ }^{1}$ Scot J. Matkovich, ${ }^{1}$ Qunying Yuan, ${ }^{2}$ Wen Zhao, ${ }^{2}$ Atsuko Yatani, ${ }^{3}$ \\ Joan Heller Brown, ${ }^{4}$ Jeffery D. Molkentin, ${ }^{5}$ Evangelia G. Kranias, ${ }^{2}$ and Gerald W. Dorn II1,2 \\ ${ }^{1}$ Center for Pharmacogenomics, Washington University in St. Louis, St. Louis, Missouri, USA. 2Department of Pharmacology and Cell Biophysics, \\ University of Cincinnati, Cincinnati, Ohio, USA. ${ }^{3}$ Department of Surgery, University of Medicine and Dentistry of New Jersey, Newark, New Jersey, USA. \\ ${ }^{4}$ Department of Pharmacology, University of California, San Diego, La Jolla, California, USA \\ 5Department of Pediatrics, Cincinnati Children's Hospital, Cincinnati, Ohio, USA.
}

\begin{abstract}
Transcriptional upregulation of the proapoptotic BCL2 family protein NIX limits red blood cell formation and can cause heart failure by inducing cell death, but the requisite molecular events are poorly defined. Here, we show complementary mechanisms for NIX-mediated cell death involving direct and ER/sarcoplasmic reticulum-mediated (ER/SR-mediated) mitochondria disruption. Endogenous cardiac NIX and recombinant NIX localize both to the mitochondria and to the ER/SR. In genetic mouse models, cardiomyocyte ER/SR calcium stores are proportional to the level of expressed NIX. Whereas Nix ablation was protective in a mouse model of apoptotic cardiomyopathy, genetic correction of the decreased SR calcium content of Nix-null mice restored sensitivity to cell death and reestablished cardiomyopathy. Nix mutants specific to ER/SR or mitochondria activated caspases and were equally lethal, but only ER/SR-Nix caused loss of the mitochondrial membrane potential. These results establish a new function for NIX as an integrator of transcriptional and calcium-mediated signals for programmed cell death.
\end{abstract}

\section{Introduction}

Apoptosis is essential for normal organism development and tissue homeostasis but creates or contributes to disease when it deviates from normal physiological constraints. Members of the BCL2 family of mitochondria-targeted proteins play central regulatory roles by positively or negatively influencing apoptotic signals from external and internal death stimuli. Permeabilization of the mitochondrial outer membrane by proapoptotic BAX and BAK is the commitment step for apoptosis because it is the first event to damage intracellular organelles (1). This gatekeeper function is regulated by $\mathrm{BH} 3$-only factors that are transcriptionally upregulated or posttranslationally activated in response to specific cell death signals. Tissue-specific expression of $\mathrm{BH} 3$-only proteins confers situational precision to apoptotic responses. Death signaling by these factors is opposed by antiapoptotic BCL2 proteins that bind to and sequester $\mathrm{BH} 3$-only proteins, thereby preventing mitochondrial outer membrane permeabilization by BAX and BAK (2).

The $\mathrm{BH} 3$-only protein NIX is expressed under specific physiological or pathological conditions in hematopoietic cells, cardiac myocytes, and other cell types. In the hematopoietic system, Nix expression increases during normal erythroblast maturation and is essential to restraining erythrocyte production and for mitochondrial pruning in maturing erythroblasts (3-5). In the heart, Nix is transcriptionally upregulated during pathological cardiac hypertrophy in response to hemodynamic overload or neurohormonal excess $(6,7)$. NIX-mediated apoptosis contributes to pro-

Conflict of interest: The authors have declared that no conflict of interest exists. Nonstandard abbreviations used: COX IV, cyclooxygenase IV; CSQN, calsequestrin; DKO, double knockout; HEK, human embryonic kidney; $\mathrm{I}_{\mathrm{Ca}}$, inward calcium current; $\Delta \psi_{\mathrm{m}}$, mitochondrial membrane potential; NCX, sodium-calcium exchanger; PLN, phospholamban; PTP, permeability transition pore; RYR, ryanodine receptor; SERCA, SR calcium ATPase; SR, sarcoplasmic reticulum; TMRE, tetramethylrhodamine ethyl ester.

Citation for this article: J. Clin. Invest. 119:203-212 (2009). doi:10.1172/JCI36445. grammed loss of cardiac myocytes that leads to heart failure (8). In each of these roles, mitochondria are central to the function of NIX. Consistent with this notion, an alternately spliced mouse NIX isoform lacking the domain that targets Nix to mitochondria has no proapoptotic activity (7).

Emerging evidence indicates that some BCL2-related factors localize to ER in addition to mitochondria (1). Major functions of ER and of analogous sarcoplasmic reticulum (SR) in the heart are storage and stimulated release of calcium. Mitochondria that are closely associated with ER/SR can capture released calcium and use it to stimulate energy production or to provoke apoptosis/necrosis, depending upon ER/SR calcium content, the magnitude of the calcium signal, and other factors (9). Overexpression of antiapoptotic BCL2 or combined ablation of proapoptotic BAX and BAK decreases ER calcium content and protects against cell demise in vitro $(10,11)$. In both instances, correction of ER calcium content by overexpression of the SR calcium ATPase (SERCA) calcium-uptake pump restores the lethality of calciummobilizing agents. Conversely, overexpression of BAX and BAK enhances ER-mitochondrial calcium transfer and increases apoptosis (12). Thus, proapoptotic BCL2 family proteins have the potential to contribute to cell death directly through effects on mitochondrial permeability and indirectly by increasing calcium delivery from ER to mitochondria.

Although tissue culture experimentation indicates that BCL2 family proteins can play a role in calcium-mediated programmed cell death, the in vivo relevance of this mechanism has not been established. We considered that an ER-mediated calcium trigger for cell death is likely to have importance in cardiac or skeletal myocytes where the proximity between ER/SR and mitochondria that are essential for maintenance of calcium-rich junctional microdomains is enforced by highly organized subcellular architecture (13). Indeed, the original observation of calcium-induced cell death was made in cardiac myocytes (14), and cytosolic or SR 
A

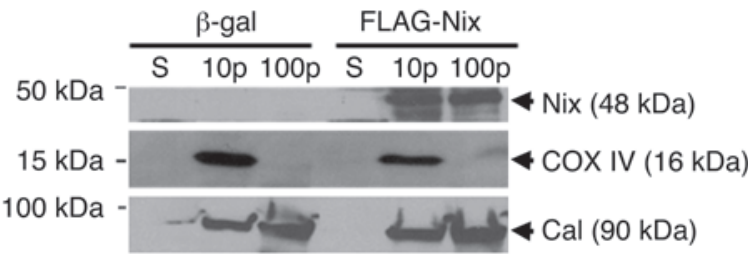

C

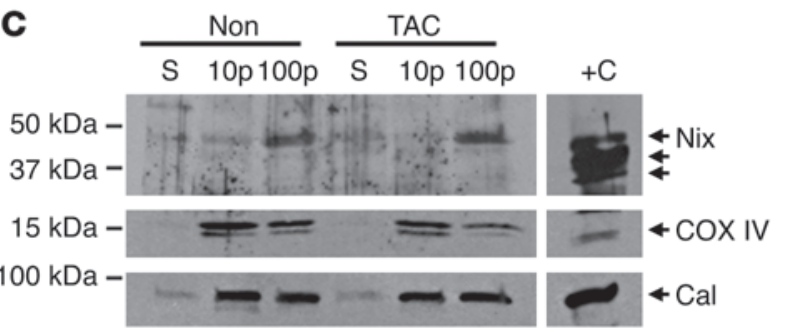

E
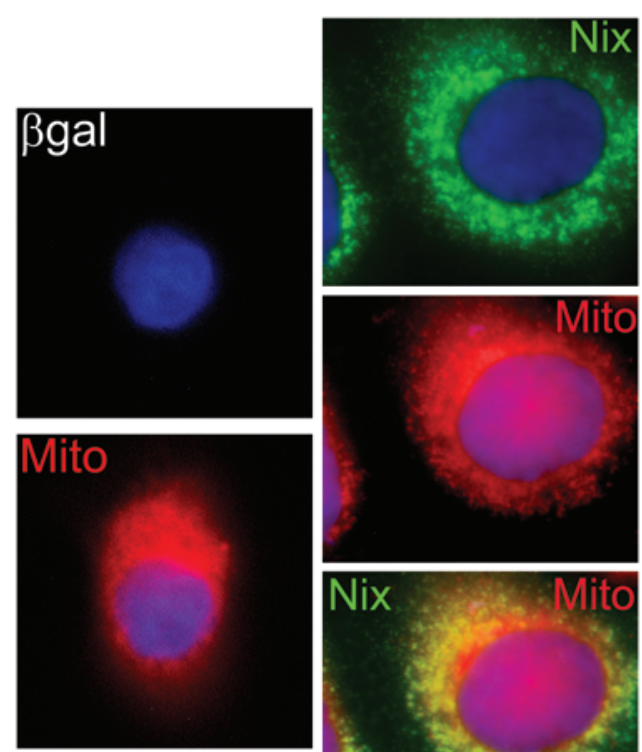

B

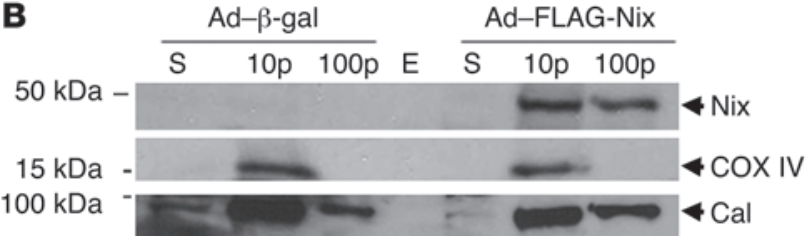

D
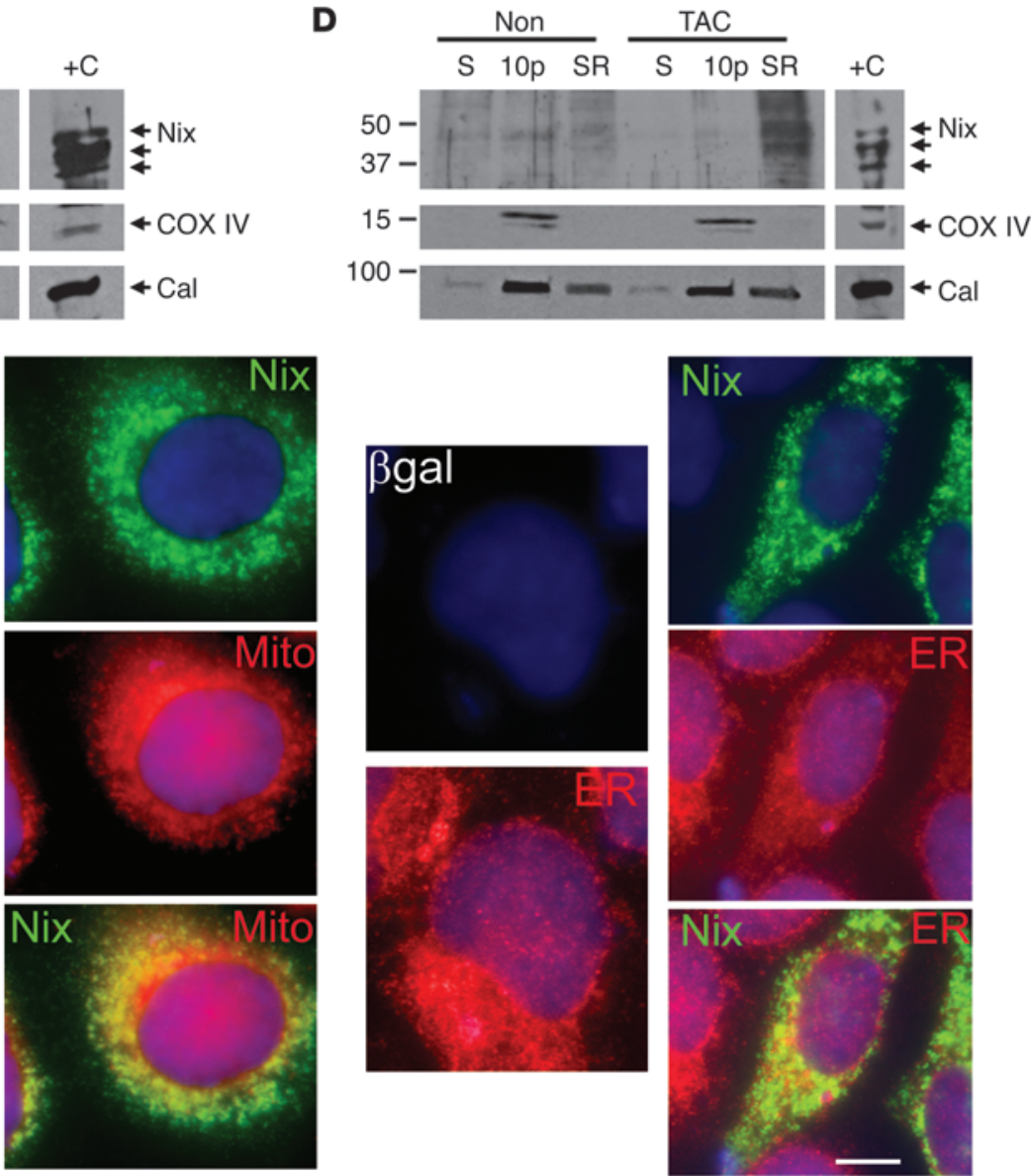

\section{Figure 1}

Nix localizes to mitochondria and ER and cofractionates with mitochondrial and ER/SR proteins. (A) HEK293 cells were transfected with FLAG-Nix or $\beta$-gal control; fractionated into $10,000 \mathrm{~g}$ pellet (10p), $100,000 \mathrm{~g}$ pellet (100p), and 100,000 $\mathrm{g}$ supernatant (S); and immunoblotted with antiFLAG, calnexin (Cal), or COX IV antibodies. (B) Neonatal rat ventricular myocytes were infected with adenoviruses encoding FLAG-Nix or $\beta$-gal control and processed as in $\mathbf{A}$. E, empty lane. (C and D) Hearts from mice subjected to 1 week of pressure overload (TAC) and nonoperated controls (Non) were fractionated into a 10,000 $\mathrm{g}$ pellet and a 100,000 $\mathrm{g}$ pellet. The 100,000 $\mathrm{g}$ pellet was separated on a discontinuous sucrose gradient to yield the SR-rich fraction (SR, see Methods). $50 \mu \mathrm{g}(\mathbf{C})$ and $20 \mu \mathrm{g}$ (D) of the indicated fractions were separated by SDS-PAGE and immunoblotted with anti-Bnip3L (Nix), calnexin, or COX IV antibodies. Positive control $(+\mathrm{C})(10 \mu \mathrm{g})$ was cellular extract from FLAG-Nix transfected HEK293 cells, showing multiple bands corresponding to Nix homodimers and heterodimers. (E) FLAG epitope-tagged Nix or $\beta$-gal control (both green) were transiently expressed in HEK293 cells and analyzed by fluorescence microscopy for colocalization with mitochondrial MitoFluor Red 589 (Mito) or ER calnexin (both red). Nuclei are blue (DAPI). Overlay for Nix is at bottom. Original magnification, $\times 1,000$. Scale bar: $10 \mu \mathrm{m}$ (shown for comparison).

calcium overload is accepted as contributing to cardiomyocyte demise in heart failure and ischemic injury $(15,16)$.

Here, using gene ablation, gene transfer, and genetic complementation approaches, we examined the contribution of ER/SR calcium to NIX-mediated cell death in vitro and to cardiac failure in vivo. We demonstrate that Nix localizes in part to the ER/SR and increases SR calcium content, which causes mitochondrial permeability transition and programmed cell death. These findings iden- tify Nix-induced modulation of SR calcium as a cell-death mechanism that promotes propagation of ER/SR-mitochondrial calcium transfer, thus conferring sensitivity to environmental stressors.

\section{Results}

NIX regulates ER calcium stores. Like most other BH3-only Bcl2 family proteins (17), NIX has a carboxyl terminus transmembrane domain that is required for mitochondrial localization and outer mem- 

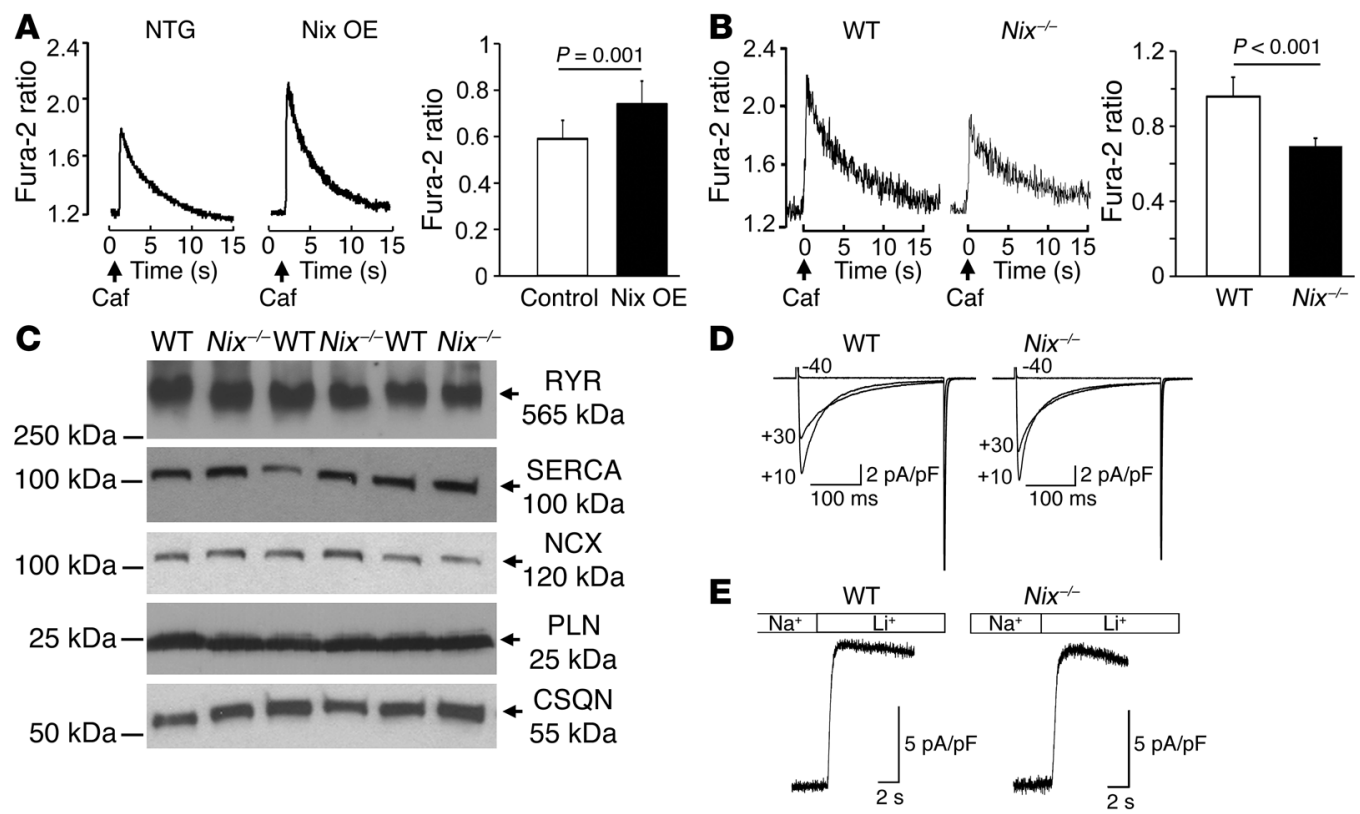

Figure 2

Nix regulates ER and SR calcium stores. (A) Ventricular cardiac myocytes isolated from nontransgenic (NTG) or conditional Nix-overexpressing (Nix OE) mouse hearts were loaded with Fura-2 AM and analyzed for caffeine-stimulated [ $\left.\mathrm{Ca}^{2+}\right]$ i by monitoring the $510 \mathrm{~nm}$ emission during rapidly alternating excitation at 340 and $380 \mathrm{~nm}$. Data are reported as the $340: 380 \mathrm{~nm}$ emission ratio. A representative pair of tracings is shown (left). Group data (right) represent mean \pm SEM of 24 NTG and 42 Nix OE cardiac myocytes from 3 pairs of hearts. Caf, caffeine. (B) Ventricular cardiac myocytes isolated from WT or Nix-knockout $\left(\mathrm{Nix}^{-1}\right)$ mouse hearts were loaded with Fura-2 AM and analyzed for caffeine-stimulated [Ca $\left.{ }^{2+}\right]$ i as above. A representative pair of tracings is shown (left). Group data (right) represent mean \pm SEM of 25 WT and 50 Nix ${ }^{-1-}$ cardiac myocytes from 5 pairs of hearts. (C) Crude cardiac extracts from Nix-null (Nix-1-) and WT hearts were subjected to immunoblotting for RYR, SERCA, NCX, PLN, and CSQN (50 $\mu \mathrm{g}$ protein/lane). (D) Representative peak $\mathrm{I}_{\mathrm{Ca}}$ traces recorded from a holding potential of $-50 \mathrm{mV}$ to the indicated test potentials in patch-clamped isolated Nix-null and WT cardiac myocytes. (E) Representative traces of $\mathrm{Na}^{+} / \mathrm{Ca}^{2+}$ exchange current induced by a rapid solution change from $150 \mathrm{mM} \mathrm{Na}^{+}$to $150 \mathrm{mM} \mathrm{Li}^{+}$(indicated above) at a holding potential of $-40 \mathrm{mV}$, recorded from Nix-null and WT cardiac myocytes.

brane permeabilization and therefore for apoptosis (7). Accordingly, recombinant NIX cofractionates with mitochondrial cyclooxygenase IV (COX IV) in transfected human embryonic kidney 293 (HEK293) cells (Figure 1A) and cultured neonatal rat ventricular cardiac myocytes (Figure 1B). As with BAX, BAK, and BCL2, however (10-12, 18), a proportion of NIX is found in subcellular fractions that are enriched in the ER/SR protein marker calnexin and have no mitochondrial contamination (COX IV) (Figure 1, A and B). Indeed, whereas endogenous myocardial NIX protein levels do not dramatically increase after pressure overloading in a conventional myocardial microsomal fraction that contains both mitochondrial and ER/SR markers (Figure 1C), sucrose gradient fractionation reveals a striking increase in ER/SR-associated NIX after transverse aortic banding (Figure 1D). In cultured HEK cells transfected with FLAGNIX, the relative proportion of NIX that is present within ER is approximately $20 \%$ of the total (Figure $1 \mathrm{E}$ ). Thus, endogenous and recombinant NIX localize, in part, to ER/SR.

We determined the in vivo consequences of ER/SR-localized NIX on intracellular calcium stores by assaying caffeine-stimulated calcium release (a measure of SR calcium content; ref. 19) in cardiac myocytes prepared from mice conditionally overexpressing Nix in the heart. Temporally defined NIX expression in adult mouse hearts does not cause the widespread apoptosis and heart failure seen with neonatal expression (20) and therefore permits characterization of NIX functions in cardiac myocytes. Isolated cardiac myocytes from conditional NIX-overexpressing mice had $25 \% \pm 5 \%$ greater increases in caffeine-stimulated cytosolic $\mathrm{Ca}^{2+}\left(\left[\mathrm{Ca}^{2+}\right]_{i}\right)$ than controls, consistent with increased SR calcium stores (Figure 2A). The reciprocal experiment, performed using cardiac myocytes isolated from mice in which Nix was genetically ablated (4), showed that the SR $\mathrm{Ca}^{2+}$ content, assessed by caffeine application, was $28 \% \pm 4 \%$ lower than in controls (Figure 2B). Levels of immunoreactive SERCA, phospholamban (PLN), ryanodine receptor (RYR), $\mathrm{Na} / \mathrm{Ca}^{2+}$ exchanger, and calsequestrin (CSQN) were unaffected by Nix ablation (Figure 2C). Likewise, the peak inward calcium current $\left(\mathrm{I}_{\mathrm{Ca}}\right)$ (Figure 2D) and sodium-calcium exchanger (NCX) current (Figure $2 \mathrm{E})$ were the same in Nix-KO and WT hearts. Thus, cardiac myocyte SR calcium stores change in the same direction as experimentally manipulated cardiac Nix expression.

Restoration of Nix-knockout SR calcium by SERCA disinhibition. Overexpression of SERCA, the ER/SR calcium-uptake pump, corrects ER calcium in cells derived from BAX/BAK double-knockout (DKO) mice (11). In the heart, SERCA is tonically inhibited by PLN (21). Therefore, to determine whether NIX-regulated SR calcium has a role in programmed cardiomyocyte death, we used a genetic complementation approach to restore SR calcium content in cardiac myocytes of Nix-knockout mice by crossing them with PLN-knockout mice (22). Disinhibition of SERCA by PLN ablation in Nix-null cardiac myocytes fully restored SR calcium stores, measured as the increase in caffeine-stimulated $\left[\mathrm{Ca}^{2+}\right]_{\mathrm{i}}$ (Figure $\left.3 \mathrm{~A}\right)$. Calcium cycling during phasic electrical stimulation, a measure of SR calcium available for excitation-contraction coupling (23), and cardiomyocyte 


\section{A}

\section{B}
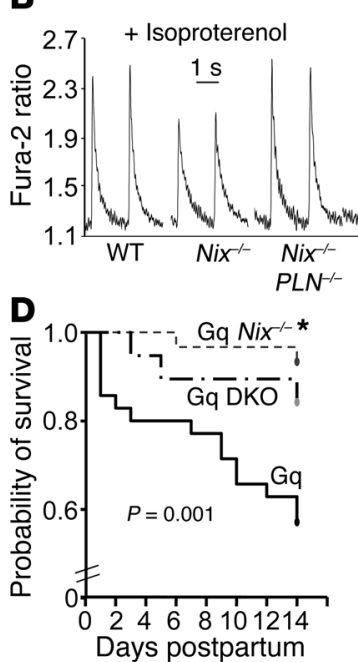
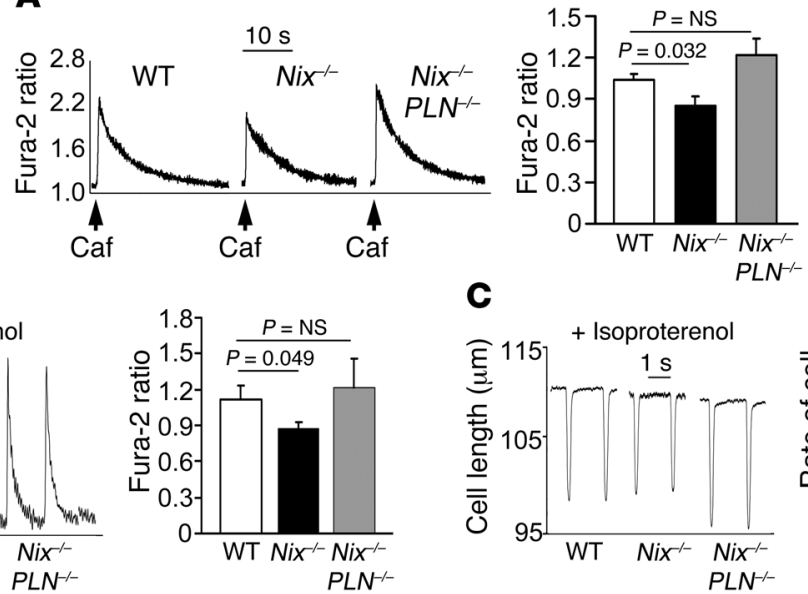

$E$

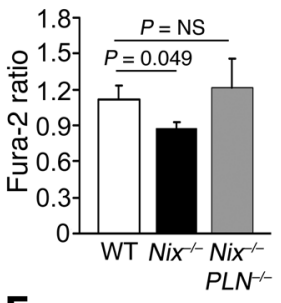

$\mathbf{F}$
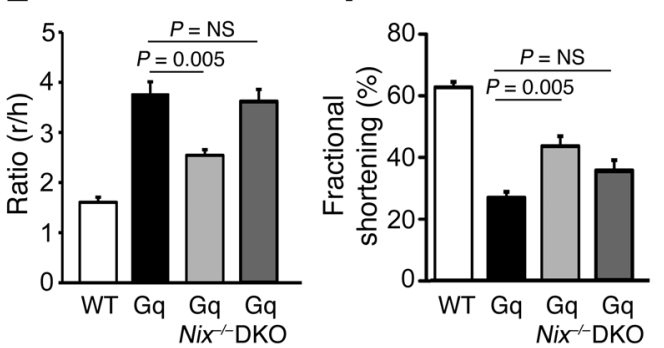

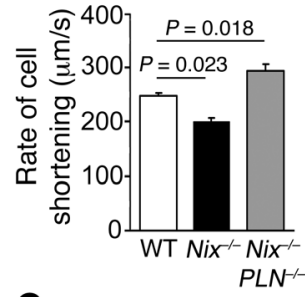

G

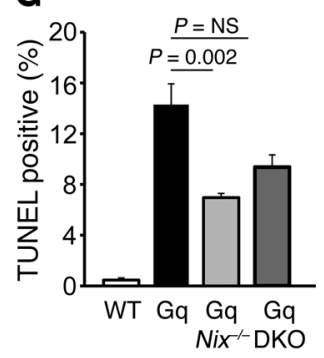

Figure 3

In vivo restoration of SR calcium stores in Nix-knockout cardiac myocytes by SERCA disinhibition reverses the Nix-null rescue of Gq peripartum cardiomyopathy. (A) Ventricular cardiac myocytes isolated from WT, Nix-knockout (Nix $\left.{ }^{-1-}\right)$, or Nix/PLN-DKO $\left(\mathrm{Nix}^{-1-} \mathrm{PLN} \mathrm{N}^{--}\right)$mouse hearts were loaded with Fura-2 AM and analyzed for caffeine-stimulated [ $\left.\mathrm{Ca}^{2+}\right]$ i. A representative set of tracings is shown (left). Group data (right) represent mean \pm SEM of $35 \mathrm{WT}, 28 \mathrm{Nix}^{-1-}$, and $38 \mathrm{Nix}^{-1-} \mathrm{PLN}^{-/-}$cardiac myocytes from $n=3$ to 4 hearts each. (B) Pacing-stimulated [Ca $\left.{ }^{2+}\right] \mathrm{in}$ ventricular myocytes from the same groups. Representative tracings are shown (left) and group data represented as mean \pm SEM (right). (C) Contraction of paced ventricular myocytes from the same groups. Representative contraction tracings are shown as the absolute change in cell length over time (left). Quantitative analysis of the peak rate of change of cell shortening are represented as mean \pm SEM (right). Horizontal bars indicate time. (D) Kaplan-Meier analysis of mouse survival in the peripartum period. Daily survival of Gq $(n=36)$, Gq Nix ${ }^{-1-}(n=30)$, and Gq Nix ${ }^{-1-P L N}-\mathrm{DKO}$ (Gq DKO, $n=19$ ) dams was assessed after giving birth. log-rank statistic was employed to detect statistical significance. ${ }^{*} P=0.017$ versus $\mathrm{Gq}$ by post-hoc test (Holms-Sidak). (E-G) Comparative analysis of left ventricular dilation (E, measured as the ratio of ventricular radius $[r]$ to wall thickness [h]), contraction (F, measured as echocardiographic percentage of fractional shortening), and apoptosis (G, measured as the percentage of TUNEL-positive cardiac myocytes) for the same study groups ( $n=6-13 / g r o u p)$. WT is shown for comparison with normal. Statistical significance was determined by 1 -way ANOVA and Tukey's post-hoc testing.

contraction itself were likewise diminished in Nix-knockout mice and fully restored by combined PLN ablation (Figure 3, B and C).

SR calcium stores contribute to Nix-mediated programmed cell death. In the heart, Nix is transcriptionally upregulated in Gq-mediated hypertrophies (6). Nix-mediated cardiomyocyte apoptosis is a key factor in hypertrophy decompensation, including the lethal peripartum heart failure that develops in cardiac Gq transgenic mice ( 7 , $8,24)$. We used this experimental model to explore the consequences of SR calcium on Nix-mediated programmed cell death and heart failure. For these experiments, the Gq transgene was introduced into the Nix-knockout (Gq-Nix-KO) and Nix/PLN-DKO (Gq-Nix/PLN-DKO) backgrounds and the postpartum courses followed in comparison with those of the mice carrying the Gq transgene alone (Gq mice).

Consistent with previous reports $(24,25)$, approximately half of peripartum $\mathrm{Gq}$ mice died of an aggressive apoptotic cardiomyopathy within 2 weeks of delivery (Figure 3D). As recently reported in a different genetic mouse strain (8), Nix ablation in Gq mice reduced peripartal mortality (Figure 3D), prevented adverse left ventricular dilation (ratio of ventricular radius [r] to wall thickness [h]; Figure $3 \mathrm{E}$ ), improved cardiac performance (percentage of echocardiographic fractional shortening; Figure 3F), and reduced cardiomyocyte apoptosis (Figure 3G). Restoration of SR calcium content by combined PLN ablation in Gq-Nix/PLN-DKO mice reversed the salutary effects of Nix ablation on ventricular dilation and contractile performance (Figure 3, E and F). Attenuation of $\mathrm{Nix}^{-/-}$protection against peripartum lethality was also observed but was limited to the first postpartum week (Figure 3D), and effects on TUNEL positivity were minimal (Figure $3 \mathrm{G}$ ). These findings indicate that the adverse effects of restoring SR calcium in $\mathrm{Nix} x^{-1-}$ mice on Gq-mediated peripartal heart failure are not entirely explained by increased cardiac myocyte apoptosis. Consistent with this conclusion, Gq-Nix/PLN-DKO peripartum mice universally exhibited evidence of cardiomyocyte necrosis in the form of dystrophic calcification (7/7), which did not appear at all in Gq-Nix-KO peripartum mice $(0 / 6)$ and which was evident in only one-third $(2 / 6)$ of $\mathrm{Gq}$ peripartum mice. These findings link SR calcium to NIX-mediated necrotic cardiomyocyte death and heart failure. 
A

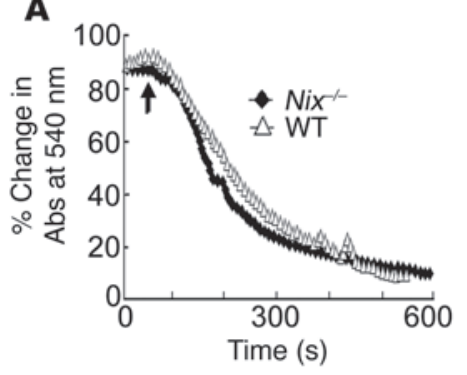

D

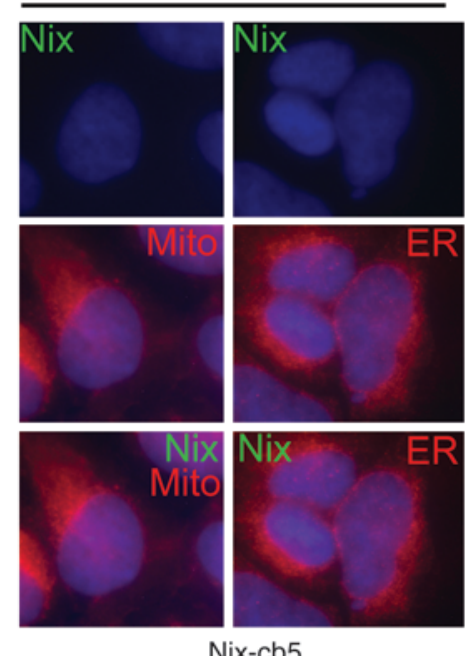

Nix-cb5
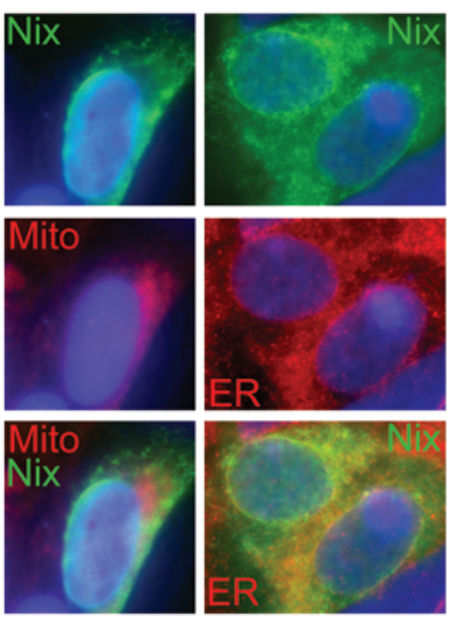

B

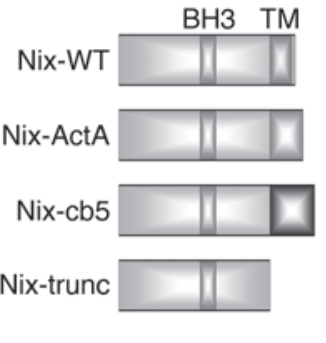

Nix-WT
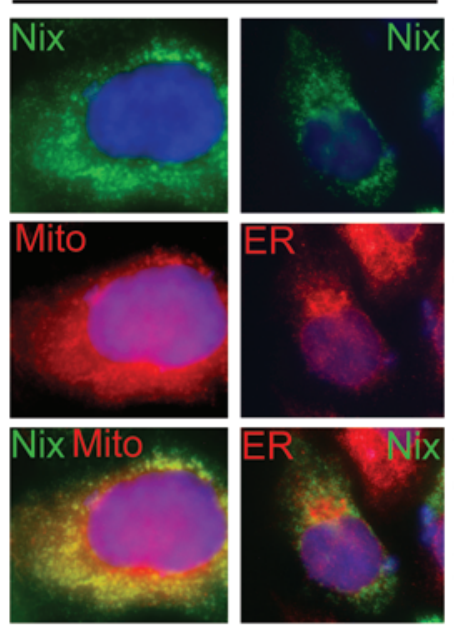

Nix-trunc
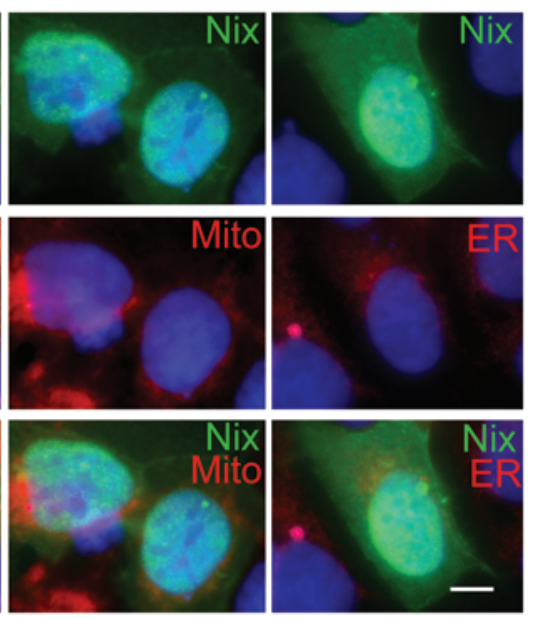

Figure 4

Creation of mitochondria- and ER-specific Nix mutants. (A) Calcium-induced swelling of purified WT (triangles) and Nix-knockout (diamonds) liver mitochondria. Calcium $(250 \mu \mathrm{M})$ was added (arrow) and the decrease in absorbance of $540 \mathrm{~nm}$ light assessed over time. Each curve represents the mean of 2 separate experiments. (B) Schematic depiction of mutation strategy for organelle-specific Nix. TM, putative transmembrane domain. (C and D) FLAG epitope-tagged WT Nix (Nix-WT), Nix-ActA, Nix-cb5, or truncated Nix (Nix-trunc) were transiently expressed in HEK293 cells, fractionated into 10,000 $\mathrm{g}$ pellet, 100,000 $\mathrm{g}$ pellet, and 100,000 $\mathrm{g}$ supernatant, and immunoblotted with anti-FLAG, calnexin, or COX IV antibodies (25 $\mathrm{g}$ protein/lane). (D) Transiently transfected WT Nix, Nix-ActA, Nix-cb5, or truncated Nix (all green) as in C were analyzed by fluorescence microscopy for colocalization with MitoFluor Red 589 or ER calnexin (both red). Nuclei are blue (DAPI). Overlays are shown at bottom. Original magnification, $\times 1,000$. Scale bar: $10 \mu \mathrm{m}$ (shown for comparison).

ER/SR-targeted NIX is as deadly as but acts differently than mitochondrial NIX. Calcium taken up by mitochondria triggers programmed cell death by opening mitochondrial permeability transition pores (PTPs) (26). Since ER/SR calcium overload activates this process by increasing calcium transfer through calcium "hot spots" to physically proximate mitochondria (13), we explored the effects of Nix on ER/SR calcium-mitochondrial signaling. We first established that Nix-null mitochondria are 
A
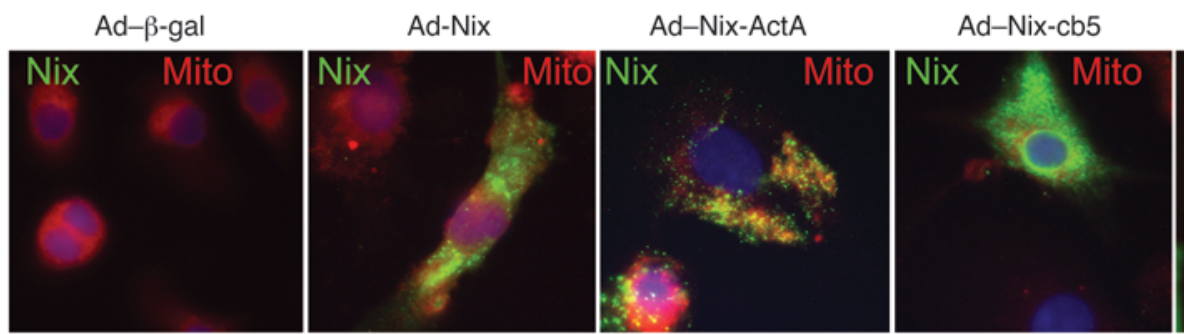

Ad-Nix-trunc

B

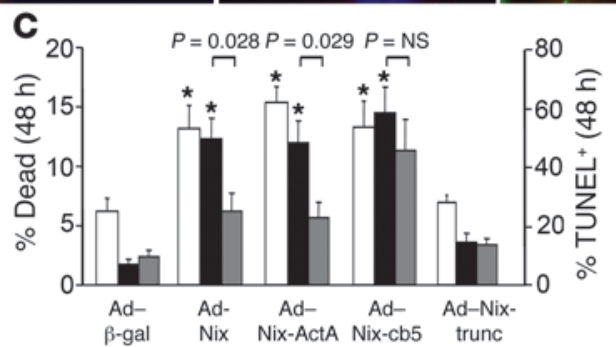

D
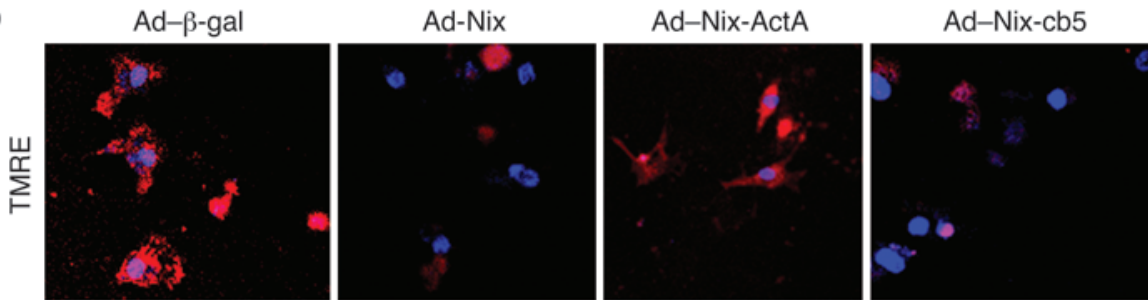

Ad-Nix-trunc
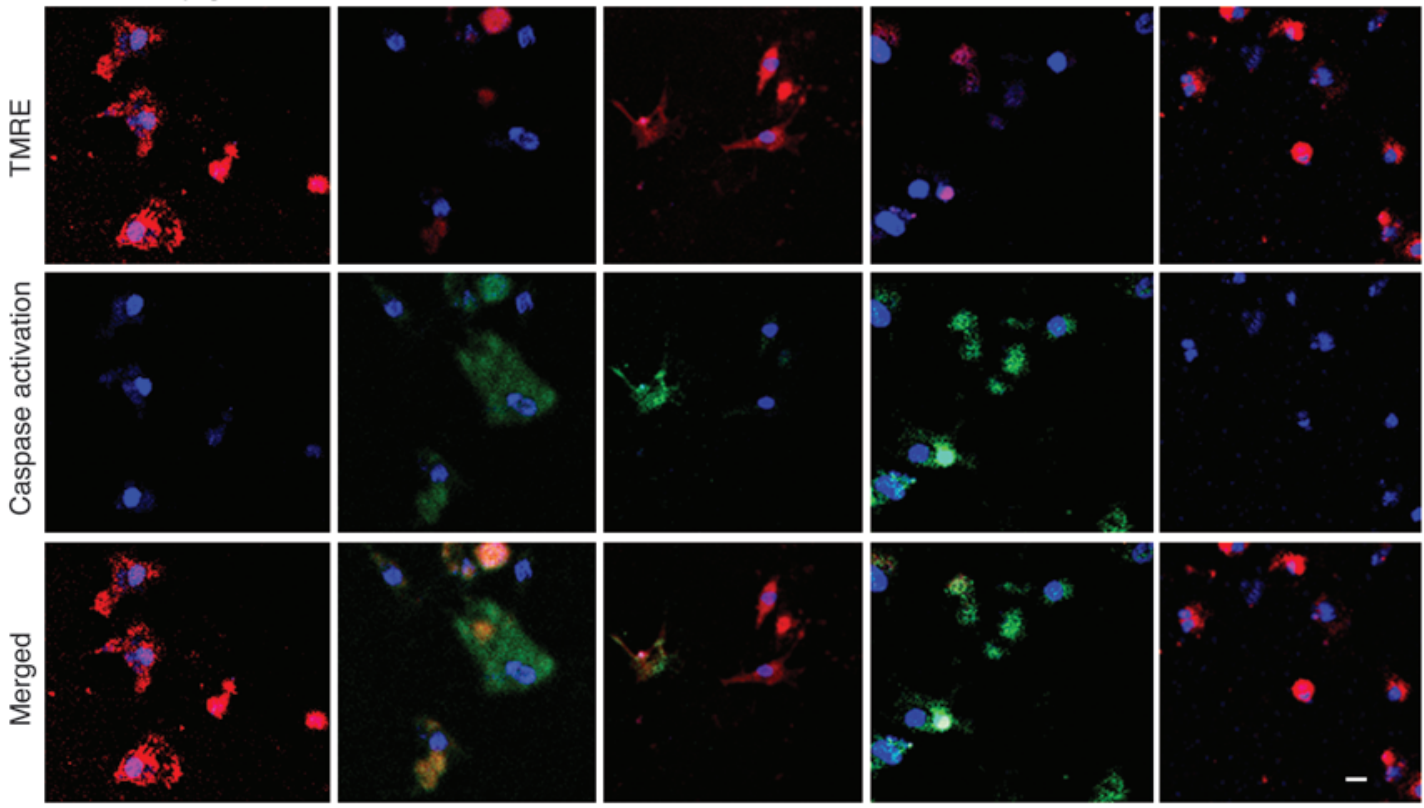

Figure 5

ER- and mitochondria-targeted Nix are equally effective in killing cultured cardiac myocytes but utilize different mediators. (A) Cultured neonatal rat cardiac myocytes were infected with adenoviruses encoding WT Nix or 1 of the 3 Nix mutants and subjected to fluorescence microscopy for subcellular localization. Shown are overlay images with FLAG-Nix (green) and MitoFluor Red 589 (red). Original magnification, $\times 1,000$. Scale bar: $10 \mu \mathrm{m}$ (shown for comparison). (B) Cultured neonatal rat cardiac myocytes were infected with adenoviruses encoding WT Nix or 1 of the 3 Nix mutants, and Nix expression was analyzed by immunoblotting for FLAG epitope and GAPDH (loading control; $25 \mu \mathrm{g} / \mathrm{lane}$ ). Arrows indicate Nix mutants with varying molecular weights. (C) Quantitative analysis of cardiomyocyte death (left $y$ axis, white bars) and TUNEL positivity in the absence (right $y$ axis, black bars) and presence (right $y$ axis, gray bars) of $25 \mu \mathrm{M}$ BAPTA-AM induced by subcellular targeting of Nix. Means \pm SEM of 4 independent experiments for death and 8 (-BAPTA) and 5 for TUNEL (+BAPTA) are shown. (D) Confocal microscopy of TMRE (red) and fluorescent caspase substrate (rhodamine 100 bis-L-aspartic acid amide; green) in cultured neonatal rat cardiac myocytes infected with adenoviruses encoding WT Nix or 1 of the 3 Nix mutants. Original magnification, $\times 400$. Nuclei are blue (Hoechst 33342). Scale bar: $20 \mu m$ (shown for comparison).

normally sensitive to calcium-mediated PTP opening (Figure $4 \mathrm{~A}$ ), indicating that NIX involvement in calcium-mediated programmed cell death is a consequence of its effect on ER/SR calcium content and not on altered mitochondrial responsiveness to $\left[\mathrm{Ca}^{2+}\right]$. We therefore directly compared the lethality of mitochondria- versus SR-associated NIX by creating NIX mutants targeted specifically to each organelle or to none at all (Figure
4B). Mitochondria and ER (or SR in heart) are physically associated, and it is therefore difficult to obtain a mitochondrial fraction that is completely free of ER/SR contamination (9, $13,27)$. However, it is possible to obtain an ER/SR-rich subcellular fraction that is essentially free of mitochondrial protein. Accordingly, immunoblot analysis using the FLAG epitope tag to localize WT and mutant NIX to fractions enriched in 


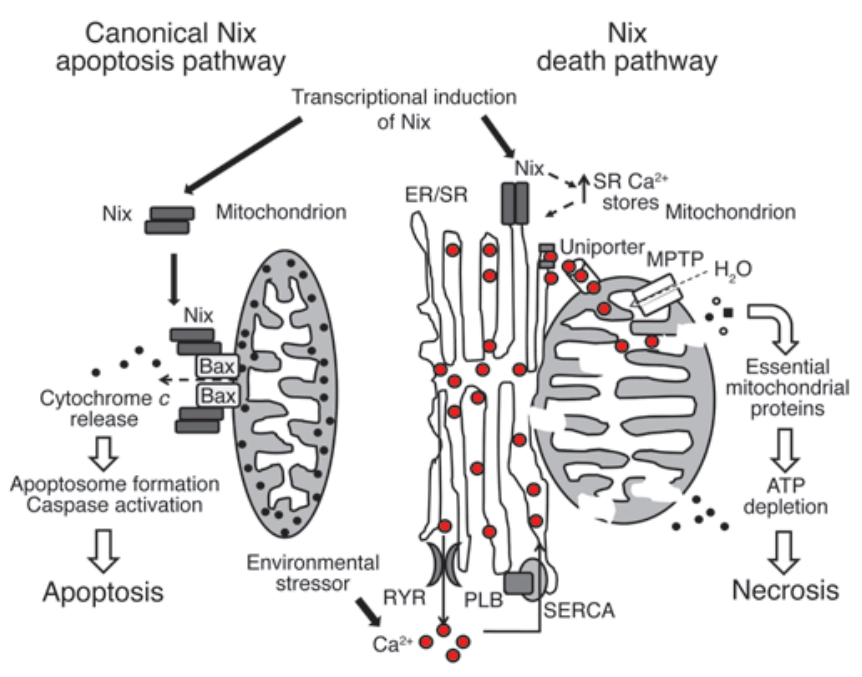

Figure 6

Mechanisms of Nix-induced cell death. Transcriptionally induced NIX is targeted to the mitochondria and ER/SR and activates programmed cell death in cells via a canonical mitochondrial pathway and what we believe is a novel ER/SR pathway. Mitochondria-targeted Nix causes mitochondrial outer membrane permeabilization, likely in coordination with Bax and Bak, leading to cytochrome $c$ release, apoptosome formation, caspase 3 activation and apoptotic cell death. ER/SR-targeted Nix contributes to ER/SR calcium overload. This sensitizes cells to environmental stimuli, resulting in local calcium release at junctional "hot spots" with mitochondria. Released calcium is taken up by a mitochondrial uniporter, causing intramitochondrial calcium overload that triggers mitochondrial permeability pore formation with loss of mitochondrial potential, mitochondrial swelling, and release of essential mitochondrial proteins, preventing ATP generation with resultant bioenergetic failure. This is rapidly followed by failure of energy-dependent, plasma membrane-localized ion transporters, causing intracellular ion overload, cellular swelling and rupture, and necrotic cell death. MPTP, mitochondrial permeability transition pore.

cytosolic, mitochondria- and ER-rich, or ER (without mitochondrial contamination) proteins revealed that NIX-ActA cofractionated with COX IV, the mitochondrial marker (Figure 4C). In contrast, NIX-cb5 cofractionated with calnexin, the ER marker (Figure 4C). Truncated NIX was seen in all fractions but was largely in the cytosolic supernatant (Figure 4C; WT NIX is also shown for comparison). Immunofluorescence microscopy of HEK293 cells transfected with each of the Nix constructs confirmed proper subcellular targeting of the mutants (Figure 4D): WT NIX (NIX-WT) colocalized with both MitoTracker Red and the ER/SR marker calnexin, whereas NIX-ActA was specific for mitochondria and NIX-cb5 was specific for ER. NIX-trunc, which lacks any targeting domain, showed a diffuse cytoplasmic and nucleoplasmic distribution (Figure 4D).

Adenoviral vectors were used for high-efficiency expression of Nix-WT and the 3 Nix mutants in cultured neonatal rat cardiac myocytes, which were then assayed for markers of apoptosis and cell viability. Organelle-specific targeting in neonatal rat cardiac myocytes was again confirmed by immunofluorescence microscopy (Figure 5A), and equivalent cardiomyocyte expression of WT and the mutant NIX proteins was shown by immunoblot analysis (Figure 5B). In studies of cell viability, TUNEL positivity, caspase activation, and mitochondrial transition pore acti- vation (tetramethylrhodamine ethyl ester [TMRE]), NIX-trunc had no effects (Figure 5, C and D). NIX-WT, NIX-ActA, and NIXcb5 exhibited similar proapoptotic activities (TUNEL positivity, Figure 5C; and caspase activation, Figure 5D), and each similarly increased cardiomyocyte death (2-fold or more; Figure 5C) compared with the control adeno- $\beta$-gal virus. However, whereas NIX-WT and NIX-cb5 caused a loss of mitochondrial membrane potential $\left(\Delta \psi_{\mathrm{m}}\right)$, indicative of PTP opening, mitochondria-specific NIX-ActA did not (Figure 5D). Since NIX does not open PTPs of isolated mitochondria (but only causes mitochondrial outer membrane permeabilization) (4), this finding reveals that Nix localization to ER/SR is essential to opening of mitochondrial PTPs. The connection between ER/SR, Nix, and programmed cell death is further supported by the observation that buffering of $\left[\mathrm{Ca}^{2+}\right]_{i}$ with the membrane-permeable $\mathrm{Ca}^{2+}$ chelator BAPTA-AM strikingly reduced apoptosis caused by Nix-WT and Nix-ActA but not by Nix-cb5 (Figure 5C). This demonstrates a unique role for compartmentalized calcium (i.e., not subject to BAPTA chelation; ref. 28) in cell death caused by the ER/SRspecific Nix mutant.

\section{Discussion}

Here, we show that the proapoptotic Bcl2 family member NIX activates a programmed cell death pathway modulated by ER/SR calcium in cardiac myocytes. NIX localizes in part to ER/SR and contributes to SR calcium overload that mediates cell death in a manner consistent with local propagation of ER/SR-mitochondrial calcium transfer. This reflects a dual mechanism for cell death mediated by Nix: transcriptional Nix upregulation leads to apoptosis through the intrinsic mitochondrial pathway, while modulation of intracellular calcium stores provides for sensitivity to environmental stressors that induce cell death via mitochondrial permeability transition (Figure 6).

A number of Bcl2 family members are expressed under transcriptional control in myocardial disease, including NIX and the closely related proapoptotic BH3-only protein BNIP3 (6-8, 29). The canonical role of these and other BH3-only factors is to sense stress signals and promote mitochondrial pore formation by the multidomain $\mathrm{Bcl} 2$ proteins $\mathrm{BAX}$ and $\mathrm{BAK}(30-32)$. Permeabilization of mitochondrial outer membranes by BAX/ BAK releases cytochrome $c$ from the mitochondrial intermembranous space and permits its interaction with cytosolic factors that initiate apoptosis signaling via the caspase cascade $(32,33)$. Cardiac-expressed antiapoptotic Bcl2 members, such as BCL2 and BCL-xl $(32,34)$, antagonize this process. Thus, BH3-only proteins are the sensors, multidomain proteins the effectors, and BCL2/BCL-xl the negative regulators of mitochondrial pathway apoptosis.

In the heart, cardiac myocyte viability and the functional and structural integrity of the injured or stressed myocardium are determined in part by regulated expression of Nix and related mitochondrial death factors. Nix mRNA is not present in measurable amounts in normal myocardium but is strikingly increased in experimental genetic and pressure overload hypertrophy and in hypertensive human hearts $(7,8)$. Nix-mediated activation of mitochondrial pathway apoptosis signaling, indicated by cytochrome $c$ release and caspase 3 cleavage, has been shown in a variety of cultured cell systems and in in vivo hypertrophied and failing myocardia $(7,8)$. Mitochondria are also central to programmed necrotic death mediated through opening of mitochondrial PTPs (26). 
Although there are reports that some BH3-only factors can cause nonapoptotic cell death (35), a specific mechanistic role for Nix or other primarily mitochondrial $\mathrm{BH} 3$-only proteins in programmed necrosis has not been described.

We observed that Nix increases ER/SR calcium and that ER/SRlocalized Nix (but not mitochondria-specific Nix) promotes mitochondrial permeability transition. It seems counterintuitive that mitochondria-targeted Nix cannot open the mitochondrial transition pore but that ER/SR-targeted Nix does so. This indicates an indirect effect of Nix on mitochondria that is mediated through the ER/SR, a notion that is supported by our previous observation that direct application of recombinant Nix protein to isolated mitochondria in a cell-free system does not cause mitochondrial swelling (a hallmark of permeability transition) (4). In contrast, calcium readily causes mitochondrial swelling in the same assay (ref. 4 and Figure 4A), and since Nix increases $\mathrm{ER} / \mathrm{SR}$ calcium, this reveals a likely mechanism: calcium can be taken up by mitochondria through the poorly characterized low-affinity calcium uniport transporter (uniporter). Physical proximity between ER/SR and mitochondria in cardiac myocytes $(27,36)$ creates junctional calcium "hot spots" at which locally high concentrations of calcium can be efficiently taken up by the uniporter (13). Calcium within these hot spots is spatially restricted and therefore protected from the conventional cyclical changes of excitation-contraction coupling and, in our and other studies, from chelation by cytosolic BAPTA (28) (see Figure 5C). Calcium delivery to the mitochondria activates matrix dehydrogenases, thus impairing mitochondrial respiration and depleting ATP necessary to sustain the electrochemical gradient across mitochondrial inner membranes. Mitochondrial depolarization and ATP depletion lead to suspension of minimal essential functions, i.e., necrotic death. The loss of $\Delta \psi_{\mathrm{m}}$ in cells expressing WT and ER/SR-targeted Nix but not mitochondria-specific Nix is indicated in our studies by decreased fluorescence of red mitochondrial TMRE staining in Figure 5D. As a consequence of increased mitochondrial inner-membrane permeability, there is an oncotic influx of water resulting in mitochondrial swelling and ultimately disruption of the mitochondrial outer membrane. Physical rupture of mitochondria due to permeability transition releases normally sequestered intermembranous proteins, cytochrome $c$, AIF, and endoG, each of which can activate apoptotic pathways (1). Thus, as shown by caspase activation and TUNEL positivity in Figure 5, apoptotic pathways can be activated indirectly by ER/SR-localized Nix as a consequence of the mitochondrial permeability transition and resulting membrane rupture $(37,38)$.

Establishing the in vivo consequences of ER/SR-targeted Nix was a major goal of these investigations, but precisely how Nix increases cardiomyocyte ER/SR calcium stores and whether Nix may be more important at either ER or SR (to the extent that they can be structurally and functionally distinguished) is not known. Regulation of ER calcium and mitochondrial death pathways has previously been described for BCL2, the multidomain proteins BAX and BAK, and the ER-specific BH3only factor BIK $(39,40)$. Overexpression of antiapoptotic Bcl2 or Bcl-xl or ablation of proapoptotic BAX and BAK reduced ER calcium content by increasing the leak of calcium through IP3 receptors and protected against cell death in vitro $(10,11$, 41-44). Recent studies have shown that antiapoptotic BCL2 and $B C L-x l$ reduce ER calcium content by binding to and sensitizing
IP3R $(44,45)$. Proapoptotic BAX and the BH3-only protein tBID antagonize this by preventing the interaction between IP3R and BCL2, which increases ER calcium and lowers the threshold for mitochondrial pathway apoptosis (41). IP3Rs are rare in cardiac myocytes and their role in cardiac homeostasis is controversial (46), so the cardiac relevance of any similar interactions between Nix and IP3R is uncertain.

The critical importance of ER/SR calcium for in vivo cardiomyocyte death was revealed in our studies through restoration of calcium stores in Nix-knockout hearts, achieved by ablating PLN. Because cardiomyocyte-free calcium concentrations increase in response to cellular stress, Nix-mediated modulation of ER/SR calcium and resulting ER/SR-mitochondrial crosstalk generates physiological inputs for programmed cell death in addition to conventional transcriptional regulation. Calcium involvement in cell death signaling may have special importance in the heart, as SR calcium overload produced by overexpression of an L-type calcium channel (LTCC) increases programmed cardiomyocyte death in vitro and in vivo $(16,36)$ and SR calcium overload in cardiac myocytes with impaired $\mathrm{Na}^{+} / \mathrm{Ca}^{2+}$ exchanger-mediated calcium extrusion stimulates apoptosis (47). Calcium-mediated stimulation of cardiomyocyte apoptotic and nonapoptotic death pathways has the potential to complicate efforts to enhance cardiac contractility and treat heart failure by increasing SR calcium, as with SERCA overexpression or PLN inhibition (48). The PLN-knockout mouse serves as an example of this double-edged sword: the baseline phenotype of the PLNknockout mouse is enhanced contractility due to increased SR calcium levels. Accordingly, ablation of PLN has been used to "rescue" some mouse models of heart failure $(22,48,49)$. However, enhanced myocyte contractility with PLN ablation has not universally been associated with improved function, and in some instances, PLN ablation has increased cardiac myocyte apoptosis (50-53). In the current studies, environmental stress in the form of pregnancy was a necessary cofactor for both programmed cardiomyocyte death induced by $\mathrm{Gq}$ overexpression and for resensitization of the cell death response in Gq-Nix KO mice after restoration of SR calcium by PLN ablation. This provides further support for the idea that Nix responds to both transcriptional (Gq transgene) and physiological (pregnancy) stressors and acts as a coincidence detector to integrate genetic and environmental cell death signals. The only other known function of Nix is to stimulate autophagic removal of mitochondria from maturing erythroblasts prior to their release into the blood stream as circulating reticulocytes $(3,5)$. Further studies will determine whether this function also requires an interaction between ER and mitochondria, but if mitochondrial pruning is analogous to "programmed mitochondrial death," it is likely that similar mechanisms are involved.

\section{Methods}

Characterization of genetically modeled mice. Mice with inducible cardiac myocyte-specific expression of Nix (20), germline ablation of Nix (4), and cardiac-specific Gq overexpression (54) were described previously. Gq transgenic mice were crossed with Nix-null (4) and PLN-null mice (22) for peripartum cardiomyopathy studies. All animal studies were approved by the Institutional Animal Care and Use Committee at the University of Cincinnati and the Animal Studies Committee at Washington University School of Medicine. 2D directed M-mode echocardiography, histopathology, and TUNEL studies were performed as described previously (8). 
Subcellular cardiac fractionation. Hearts from mice were subjected to 7 days of pressure overload by transverse aortic constriction (8) (with transcoarctation gradients of $81 \pm 3 \mathrm{mmHg}$ ), snap-frozen in liquid nitrogen, and homogenized in a buffer containing $10 \mathrm{mM}$ HEPES, $\mathrm{pH}$ 7.2, $320 \mathrm{mM}$ sucrose, $3 \mathrm{mM} \mathrm{MgCl}_{2}$, $25 \mathrm{mM} \mathrm{Na}_{2} \mathrm{P}_{4} \mathrm{O}_{7}, 1 \mathrm{mM}$ DTT, $5 \mathrm{mM}$ EGTA, $1 \mathrm{mM}$ PMSF, and Complete Mini Protease Inhibitor Cocktail Tablet (Roche) (55). The homogenate was filtered through a 40-micron filter and subjected to centrifugation at $3,800 \mathrm{~g}$ for 15 minutes to remove nuclei and myofibrils. The supernatant was subjected to centrifugation at $10,000 \mathrm{~g}$ for 20 minutes, and the resultant pellet was labeled $10 \mathrm{p}$. The supernatant was then subjected to centrifugation at $100,000 \mathrm{~g}$ for 1 hour. The supernatant from this was labeled as the $\mathrm{S}$ fraction. The resultant pellet (100p) was resuspended and subjected to separation on a discontinuous sucrose gradient (15\%-35\%) at $132,000 \mathrm{~g}$ for 16.5 hours. The microsomal enriched (SR) fraction was isolated from the $15 \% / 35 \%$ interface. The various fractions were then subjected to immunoblotting for Nix (anti-Bnip3L antibody; Abcam).

Studies in fibroblasts. HEK cells were transfected with N-terminal FLAGtagged mouse Nix (7), and subcellular fractionation was performed as described (55). Immunoblotting was performed with antibodies against FLAG (M2 monoclonal antibody; Sigma-Aldrich), COX IV (Abcam), calnexin (Santa Cruz Biotechnology Inc.), and GAPDH (Abcam).

Isolated myocyte mechanics and $\mathrm{Ca}^{2+}$ kinetics. Isolation of mouse ventricular myocytes was carried out as described previously (56). Cell shortening and Ca transients were measured separately from cardiomyocytes at room temperature. Myocytes were field stimulated by a Grass S5 stimulator $(0.5 \mathrm{~Hz}$, square waves) in the absence or presence of $0.1 \mu \mathrm{M}$ isoproterenol, and contractions were videotaped and digitized on a computer. For Ca signal measurements, cells were loaded with Fura-2 (Fura-2 /AM; $2 \mu \mathrm{M}$ ) and alternately excited at 340 and $380 \mathrm{~nm}$ by a Delta Scan dual-beam spectrophotofluorometer (Photon Technology International) at baseline conditions and upon rapid application of $10 \mathrm{mM}$ caffeine. Ca transients were recorded as the $340 / 380 \mathrm{~nm}$ ratio of the resulting $510 \mathrm{~nm}$ emissions in the absence or presence of $0.1 \mu \mathrm{M}$ isoproterenol (Sigma-Aldrich). Data were analyzed by Felix software (Photon Technology International). Peak $\mathrm{I}_{\mathrm{Ca}}$ and NCX current were measured using patch clamp technique in isolated cardiac myocytes, as described (57).

Analysis of calcium-handling proteins. Frozen cardiac tissues were homogenized in $50 \mathrm{mM}$ Tris, $\mathrm{pH}$ 8.0, $1 \mathrm{mM}$ EDTA, $5 \mathrm{mM} \mathrm{NaN3}$, and 1 Complete Mini Protease Inhibitor Tablet (Roche) and cleared of undigested matter with filtration (40 micron) and centrifugation at 1,000 $\mathrm{g}$ for 15 minutes. The resultant crude cardiac extracts were immunoblotted for RYR, SERCA, NCX, PLN, and CSQN (all from Affinity BioReagents).

Construction and characterization of Nix mutants. Employing an N-terminal FLAG-tagged mouse Nix cDNA described previously (7), we used PCR-based strategies to obtain the following constructs: Nix truncated at amino acid 186, immediately prior to the transmembrane C-terminal domain (Nix-trunc); Nix-trunc with the peptide tag ActA replacing the transmembrane domain (Nix-ActA, mitochondrially targeted mutant); and Nix-trunc with the peptide tag cb5 (Nix-cb5, ER/SR targeted mutant), as described (58). In brief, the Act $\mathrm{A}$ and cb5 amino sequences were backtranslated, C-terminal stop codons were added, and synthetic oligonucleotides corresponding to sense and antisense strands (ActA, sense strand only: 5'-CTGATCCTGGCCATGCTGGCCATCGGCGTGTTCAGCCTGGGCGCCTTCATCAAGATCATCCAGCTGAGGAAGAACAAC-3'; cb5, sense strand only: 5'-ATCACCACCGTGGAGAGCAACAGCAGCTGGTGGACCAACTGGGTGATCCCCGCCATCAGCGCCCTGGTGGTGGCCCTGATGTACAGGCTGTACATGGCCGAGGAC-3') were annealed and cloned into pBluescript between EcoRI and HindIII sites. Coding sequences for ActA+stop and cb5+stop were seamlessly joined to Nix- trunc using the technique of splicing overlap extension (59). To assess subcellular targeting of Nix constructs, HEK cells on chamber slides were transfected with Nix cDNA using FuGENE (Roche). After 24 hours, cells were fixed in $4 \%$ paraformaldehyde in PBS, permeabilized at room temperature with $0.2 \%$ Triton X-100 in PBS for 5 minutes, and treated with $0.1 \mathrm{M}$ glycine, $\mathrm{pH} 3.5$, to quench residual paraformaldehyde. Slides were blocked in $1 \%$ BSA, $0.1 \%$ Tween-20, $0.05 \% \mathrm{NaN}_{3}$ in PBS, and $3 \%$ donkey serum (Santa Cruz Biotechnology Inc.), and double antibody staining of FLAG epitopes and calnexin was performed in blocking solution with $1 \%$ donkey serum with the same antibodies used for immunoblotting studies, together with donkey anti-mouse Alexa Fluor 488 and donkey anti-mouse Alexa Fluor 594 secondary antibodies (Molecular Probes; Invitrogen). For mitochondrial staining, $200 \mathrm{nM}$ MitoFluor Red 589 was applied after antibody treatments. Slides were mounted with VECTASHIELD containing $1.5 \mu \mathrm{g} / \mathrm{ml}$ DAPI (Vector Laboratories). Images were obtained with an Olympus $\times 100$ oil-immersion lens mounted on a Nikon inverted fluorescence microscope.

Mitochondrial isolation and analysis. Liver mitochondria were isolated and pore opening determined by mitochondrial swelling assay, as described (26).

Adenoviral infection studies. Recombinant adenoviruses were created by cloning FLAG-tagged Nix (7) and mutant Nix proteins (see above) into pAdEasy-1 vector (Stratagene) using pShuttleCMV and recombination in BJ5183-AD-1 cells, according to the manufacturer's instructions. Viral sequences were confirmed by restriction digests, transfected into and amplified in HEK cells, and titrated by agarose overlay, as previously described (24). Neonatal rat ventricular myocytes were isolated from 1- to 2-day-old Sprague-Dawley rat pups and infected with adenoviruses at a titer of 100 PFUs/cell for 48 hours at $37^{\circ} \mathrm{C}$, as described previously (24). The LIVE/DEAD Assay (Invitrogen) and DeadEnd Fluorometric TUNEL kit (Promega) were employed to study cell death.

Confocal microscopy. $\Delta \psi_{\mathrm{m}}$ (to assess mitochondrial PTP formation) and caspase activation were analyzed by TMRE (26) and caspase substrate (Rhodamine 110 bis-L-aspartic acid amide; Molecular Probes, Invitrogen) immunofluorescence, respectively, by confocal microscopy performed on a Zeiss LSM 510 NLO Two-Photon Microscope with a $\times 40$ C-Apochromat objective. Lasers employed for excitation of fluorescence were $\operatorname{Ar}(458,477$, 488, and $514 \mathrm{~nm}$ ), green $\mathrm{HeNe}(543 \mathrm{~nm})$, and red $\mathrm{HeNe}(633 \mathrm{~nm})$; and images were analyzed with Aim Image Browser (Zeiss). Hoechst 33342 dye (Molecular Probes; Invitrogen) fluorescence was employed to counterstain nuclei.

Statistics. Results are mean \pm SEM. Experimental groups were compared using 2-tailed Student's $t$ test for comparison between 2 groups and 1-way ANOVA for comparison among multiple groups followed by Tukey's post-hoc test. Nonparametric testing was employed when data were not normally distributed. Dunn's post-hoc test was employed after ANOVA on ranks. log-rank test was employed for survival analyses. $P<0.05$ was considered significant.

\section{Acknowledgments}

This study was supported by National Heart, Lung, and Blood Institute grants HL059888, HL077101, and HL080008 (to G.W. Dorn), the American Heart Association (Scientist Development Grant to A. Diwan), and the Department of Veterans Affairs.

Received for publication June 10, 2008, and accepted in revised form October 15, 2008.

Address correspondence to: Gerald W. Dorn II, Washington University Center for Pharmacogenomics, 660 S. Euclid Avenue, Campus Box 8086, St. Louis, Missouri 63110, USA. Phone: (314) 362-8901; Fax: (314) 362-0186; E-mail: gdorn@dom.wustl.edu. 
1. Danial, N.N., and Korsmeyer, S.J. 2004. Cell death: critical control points. Cell. 116:205-219.

2. Gross, A., McDonnell, J.M., and Korsmeyer, S.J. 1999. BCL-2 family members and the mitochondria in apoptosis. Genes Dev. 13:1899-1911.

3. Sandoval, H., et al. 2008. Essential role for Nix in autophagic maturation of erythroid cells. Nature. 454:232-235

4. Diwan, A., et al. 2007. Unrestrained erythroblast development in $\mathrm{Nix}^{-/}$mice reveals a mechanism for apoptotic modulation of erythropoiesis. Proc. Natl. Acad. Sci. U. S. A. 104:6794-6799.

5. Schweers, R.L., et al. 2007. NIX is required for programmed mitochondrial clearance during reticulocyte maturation. Proc. Natl. Acad.Sci. U. S. A. 104:19500-19505.

6. Galvez, A.S., et al. 2006. Distinct pathways regulate proapoptotic Nix and BNip3 in cardiac stress. J. Biol. Chem. 281:1442-1448.

7. Yussman, M.G., et al. 2002. Mitochondrial death protein Nix is induced in cardiac hypertrophy and triggers apoptotic cardiomyopathy. Nat. Med. 8:725-730.

8. Diwan, A., et al. 2008. Nix-mediated apoptosis links myocardial fibrosis, cardiac remodeling, and hypertrophy decompensation. Circulation. 117:396-404.

9. Demaurex, N., and Distelhorst, C. 2003. Cell biology. Apoptosis - the calcium connection. Science. 300:65-67.

10. Foyouzi-Youssefi, R., et al. 2000. Bcl-2 decreases the free $\mathrm{Ca} 2+$ concentration within the endoplasmic reticulum. Proc. Natl. Acad. Sci. U. S. A. 97:5723-5728.

11. Scorrano, L., et al. 2003. BAX and BAK regulation of endoplasmic reticulum Ca2+: a control point for apoptosis. Science. 300:135-139.

12. Nutt, L.K., et al. 2002. Bax and Bak promote apoptosis by modulating endoplasmic reticular and mitochondrial Ca2+ stores. J. Biol. Chem. 277:9219-9225.

13. Rizzuto, R., Duchen, M.R., and Pozzan, T. 2004. Flirting in little space: the $\mathrm{ER} /$ mitochondria Ca2+ liaison. Sci. STKE. 2004:re1.

14. Fleckenstein, A., Janke, J., Doring, H.J., and Leder, O. 1974. Myocardial fiber necrosis due to intracellular Ca overload-a new principle in cardiac pathophysiology. Recent Adv. Stud. Cardiac. Struct. Metab. 4:563-580.

15. Bolli, R., and Marban, E. 1999. Molecular and cellular mechanisms of myocardial stunning. Physiol. Rev. 79:609-634

16. Nakayama, H., et al. 2007. Ca2+- and mitochondrial-dependent cardiomyocyte necrosis as a primary mediator of heart failure. J. Clin.Invest. 117:2431-2444.

17. Strasser, A. 2005. The role of BH3-only proteins in the immune system. Nat. Rev. Immunol. 5:189-200.

18. Pinton, P., et al. 2001. The Ca2+ concentration of the endoplasmic reticulum is a key determinant of ceramide-induced apoptosis: significance for the molecular mechanism of Bcl-2 action. EMBO J. 20:2690-2701

19. Su, J.Y., and Hasselbach, W. 1984. Caffeine-induced calcium release from isolated sarcoplasmic reticulum of rabbit skeletal muscle. Pflugers Arch. 400:14-21.

20. Syed, F., et al. 2004. Physiological growth synergizes with pathological genes in experimental cardiomyopathy. Circ. Res. 95:1200-1206.

21. Kranias, E.G., and Solaro, R.J. 1982. Phosphorylation of troponin I and phospholamban during catecholamine stimulation of rabbit heart. Nature. 298:182-184.

22. Luo, W., et al. 1994. Targeted ablation of the phospholamban gene is associated with markedly enhanced myocardial contractility and loss of betaagonist stimulation. Circ. Res. 75:401-409.
23. Bers, D.M. 2002. Cardiac excitation-contraction coupling. Nature. 415:198-205.

24. Adams, J.W., et al. 1998. Enhanced Galphaq signaling: a common pathway mediates cardiac hypertrophy and apoptotic heart failure. Proc. Natl. Acad. Sci. U. S. A. 95:10140-10145.

25. Hayakawa, Y., et al. 2003. Inhibition of cardiac myocyte apoptosis improves cardiac function and abolishes mortality in the peripartum cardiomyopathy of Galpha(q) transgenic mice. Circulation. 108:3036-3041.

26. Baines, C.P., et al. 2005. Loss of cyclophilin D reveals a critical role for mitochondrial permeability transition in cell death. Nature. 434:658-662.

27. Rusinol, A.E., Cui, Z., Chen, M.H., and Vance, J.E. 1994. A unique mitochondria-associated membrane fraction from rat liver has a high capacity for lipid synthesis and contains pre-Golgi secretory proteins including nascent lipoproteins. J. Biol. Chem. 269:27494-27502.

28. Szalai, G., Csordas, G., Hantash, B.M., Thomas, A.P., and Hajnoczky, G. 2000. Calcium signal transmission between ryanodine receptors and mitochondria. J. Biol. Chem. 275:15305-15313.

29. Regula, K.M., Ens, K., and Kirshenbaum, L.A. 2002. Inducible expression of BNIP3 provokes mitochondrial defects and hypoxia-mediated cell death of ventricular myocytes. Circ. Res. 91:226-231.

30. Bouillet, P., and Strasser, A. 2002. BH3-only proteins - evolutionarily conserved proapoptotic Bcl-2 family members essential for initiating programmed cell death. J. Cell Sci. 115:1567-1574.

31. Kubli, D.A., Ycaza, J.E., and Gustafsson, A.B. 2007. Bnip3 mediates mitochondrial dysfunction and cell death through Bax and Bak. Biochem. J. 405:407-415.

32. Zong, W.X., Lindsten, T., Ross, A.J., MacGregor, G.R., and Thompson, C.B. 2001. BH3-only proteins that bind pro-survival $\mathrm{Bcl}-2$ family members fail to induce apoptosis in the absence of Bax and Bak. Genes Dev. 15:1481-1486.

33. Wei, M.C., et al. 2001. Proapoptotic BAX and BAK a requisite gateway to mitochondrial dysfunction and death. Science. 292:727-730.

34. Cheng, E.H., et al. 2001. BCL-2, BCL-X(L) sequester $\mathrm{BH} 3$ domain-only molecules preventing BAX-and BAK-mediated mitochondrial apoptosis. Mol. Cell. 8:705-711.

35. Vande, V.C., et al. 2000. BNIP3 and genetic control of necrosis-like cell death through the mitochondrial permeability transition pore. Mol. Cell. Biol. 20:5454-5468

36. Chen, X., et al. 2005. Ca2+ influx-induced sarcoplasmic reticulum $\mathrm{Ca} 2+$ overload causes mitochondrial-dependent apoptosis in ventricular myocytes. Circ. Res. 97:1009-1017.

37. Gogvadze, V., Robertson, J.D., Zhivotovsky, B., and Orrenius, S. 2001. Cytochrome c release occurs via $\mathrm{Ca} 2+$-dependent and $\mathrm{Ca} 2+$-independent mechanisms that are regulated by Bax. J. Biol. Chem. 276:19066-19071.

38. Oakes, S.A., Opferman, J.T., Pozzan, T., Korsmeyer, S.J., and Scorrano, L. 2003. Regulation of endoplasmic reticulum $\mathrm{Ca} 2+$ dynamics by proapoptotic BCL-2 family members. Biochem. Pharmacol. 66:1335-1340.

39. Germain, M., and Shore, G.C. 2003. Cellular distribution of Bcl-2 family proteins. Sci. STKE. 2003:pe10.

40. Mathai, J.P., Germain, M., and Shore, G.C. 2005. $\mathrm{BH} 3$-only BIK regulates BAX,BAK-dependent release of $\mathrm{Ca} 2+$ from endoplasmic reticulum stores and mitochondrial apoptosis during stress-induced cell death. J. Biol. Chem. 280:23829-23836.

41. Oakes, S.A., et al. 2005. Proapoptotic BAX and BAK regulate the type 1 inositol trisphosphate receptor and calcium leak from the endoplasmic reticulum.
Proc. Natl. Acad. Sci. U. S. A. 102:105-110,

42. Palmer, A.E., Jin, C., Reed, J.C., and Tsien, R.Y. 2004. $\mathrm{Bcl}-2$-mediated alterations in endoplasmic reticulum $\mathrm{Ca} 2+$ analyzed with an improved genetically encoded fluorescent sensor. Proc. Natl. Acad. Sci. U. S. A. 101:17404-17409.

43. Pinton, P., et al. 2000. Reduced loading of intracellular $\mathrm{Ca}(2+)$ stores and downregulation of capacitative $\mathrm{Ca}(2+)$ influx in $\mathrm{Bcl}$-2-overexpressing cells. J. Cell Biol. 148:857-862.

44. White, C., et al. 2005. The endoplasmic reticulum gateway to apoptosis by $\mathrm{Bcl}-\mathrm{X}(\mathrm{L})$ modulation of the InsP3R. Nat. Cell Biol. 7:1021-1028.

45. Chen, R., et al. 2004. Bcl-2 functionally interacts with inositol 1,4,5-trisphosphate receptors to regulate calcium release from the ER in response to inositol 1,4,5-trisphosphate. J.Cell Biol. 166:193-203.

46. Woodcock, E.A., and Matkovich, S.J. 2005. Ins $(1,4,5) \mathrm{P}-3$ receptors and inositol phosphates in the heart - evolutionary artefacts or active signal transducers? Pharmacology \& Therapeutics 107:240-251.

47. Miyamoto, S., Howes, A.L., Adams, J.W., Dorn, G.W., and Brown, J.H. 2005. Ca2+ dysregulation induces mitochondrial depolarization and apoptosis: role of $\mathrm{Na}+\mathrm{Ca} 2+$ exchanger and AKT. J. Biol. Chem. 280:38505-38512.

48. Chien, K.R. 2000. Meeting Koch's postulates for calcium signaling in cardiac hypertrophy. J. Clin. Invest. 105:1339-1342.

49. Minamisawa, S., et al. 1999. Chronic phospholamban-sarcoplasmic reticulum calcium ATPase interaction is the critical calcium cycling defect in dilated cardiomyopathy. Cell. 99:313-322.

50. Cross, H.R., Kranias, E.G., Murphy, E., and Steenbergen, C. 2003. Ablation of PLB exacerbates ischemic injury to a lesser extent in female than male mice: protective role of NO. Am. J. Physiol. Heart Circ.Physiol. 284:H683-H690.

51. Delling, U., Sussman, M.A., and Molkentin, J.D. 2000. Re-evaluating sarcoplasmic reticulum function in heart failure. Nat. Med. 6:942-943.

52. Song, Q., et al. 2003. Rescue of cardiomyocyte dysfunction by phospholamban ablation does not prevent ventricular failure in genetic hypertrophy. J. Clin. Invest. 111:859-867.

53. Yang, Y., et al. 2006. Calmodulin kinase II inhibition protects against myocardial cell apoptosis in vivo. Am. J. Physiol. Heart Circ. Physiol. 291:H3065-H3075.

54. D’Angelo, D.D., et al. 1997. Transgenic Galphaq overexpression induces cardiac contractile failure in mice. Proc. Natl. Acad. Sci. U. S. A. 94:8121-8126.

55. Bare, D.J., Kettlun, C.S., Liang, M., Bers, D.M., and Mignery, G.A. 2005. Cardiac type 2 inositol 1,4,5trisphosphate receptor - Interaction and modulation by calcium/calmodulin-dependent protein kinase II. J. Biol. Chem. 280:15912-15920.

56. Yatani, A., Frank, K., Sako, H., Kranias, E.G., and Dorn, G.W. 1999. Cardiac-specific overexpression of Galphaq alters excitation-contraction coupling in isolated cardiac myocytes. J. Mol. Cell Cardiol. 31:1327-1336.

57. Mitarai, S., Reed, T.D., and Yatani, A. 2000. Changes in ionic currents and beta-adrenergic receptor signaling in hypertrophied myocytes overexpressing G alpha(q). Am. J. Physiol. Heart Circ. Physiol. 279:H139-H148.

58. Zhu, W., et al. 1996. Bcl-2 mutants with restricted subcellular location reveal spatially distinct pathways for apoptosis in different cell types. EMBO J. 15:4130-4141.

59. Warrens, A.N., Jones, M.D., and Lechler, R.I. 1997. Splicing by overlap extension by PCR using asymmetric amplification: an improved technique for the generation of hybrid proteins of immunological interest. Gene. 186:29-35. 